Journal Club

Editor's Note: These short, critical reviews of recent papers in the Journal, written exclusively by graduate students or postdoctoral fellows, are intended to summarize the important findings of the paper and provide additional insight and commentary. For more information on the format and purpose of the Journal Club, please see http://www.jneurosci.org/misc/ifa_features.shtml.

\title{
Deconstructing the Ability to Move to a Beat
}

\author{
Michael J. Hove ${ }^{1}$ and Michael Schwartze ${ }^{2}$ \\ ${ }^{1}$ Harvard Medical School, Boston, Massachusetts 02115, and ${ }^{2}$ School of Psychological Sciences, University of Manchester, Manchester, M13 9PL, United \\ Kingdom \\ Review of Tierney and Kraus
}

Even in its simplest form, the human ability to temporally align movements to sensory events presents a complex challenge for neuroscience. Such sensorimotor synchronization requires many interconnected processes. It depends on precise sensory encoding, rhythmic motor control, temporal prediction of upcoming events, integration of perception and action, error correction to compensate for discrepancies, and typically attention to the task. Only the interplay of these processes allows accurate synchronization, and each process has been the subject of extensive theoretical and empirical work (Repp, 2005; Repp and $\mathrm{Su}, 2013)$. However, despite significant overall progress, relatively little is known about the neural underpinnings of individual differences in synchronization abilities and how these relate to other basic neurocognitive functions.

In a recent paper in The Journal of Neuroscience, Tierney and Kraus (2013b) provide evidence for a link between the ability to synchronize movements to an auditory beat and the consistency of neural responses to speech. A large group of adolescents $(n=124)$ participated in a two-part experiment. In

Received Nov. 20, 2013; revised Dec. 20, 2013; accepted Dec. 30, 2013.

Correspondence can be addressed to either of the following: Dr. Michael J. Hove, Harvard Medical School, MGH / Martinos Center for Biomedical Imaging, 149 Thirteenth Street, Room 2660, Charlestown, MA 02129, E-mail: michaeljhove@gmail.com; or Dr. Michael Schwartze, Zochonis Building, University of Manchester, Brunswick Street, Manchester, M13 9PL, UK, E-mail: michael.schwartze@manchester.ac.uk.

DOI:10.1523/JNEUROSCI.4880-13.2014

Copyright $\odot 2014$ the authors $\quad 0270-6474 / 14 / 342403-03 \$ 15.00 / 0$ the behavioral portion, participants were asked to tap their finger in synchrony with drum sounds presented isochronously at rates of 1.5 and $2 \mathrm{~Hz}$. The primary variable of interest was the variability of the tapping rate. In the neurophysiological portion, participants were presented with a synthesized syllable (/da/, $170 \mathrm{~ms})$ at a rate of $4.35 \mathrm{~Hz}$. Participants' neural responses were recorded via auditory brainstem electroencephalography (EEG). The primary variable of interest was the consistency of the complex auditory brainstem response (cABR), which originates from the inferior colliculus and provides an index of sound-encoding quality in the auditory system.

Results indicated that subjects with lower synchronized tapping variability had more consistent brainstem encoding of the speech sounds. More specifically, tapping variability correlated with the consistency of brainstem encoding during the syllable's formant transition (5-60 ms post-onset), but not during the following steady-state vowel portion (60-170 ms). This link between synchronized tapping and early auditory encoding in the brainstem suggests an important role of the inferior colliculus "in the transformation of periodicity in auditory stimuli to motor output" (Tierney and Kraus, 2013b, p 14984). Accordingly, the authors conclude that "timing jitter within the inferior colliculus leads to a more variable representation of stimulus periodicity, and in turn, more vari- able motor output" (Tierney and Kraus, 2013b, p 14984).

Other recent studies corroborate the importance of temporal encoding for accurate synchronized tapping. As the authors note, fine temporal resolution in the auditory system likely contributes to better synchronization with auditory beeps than visual flashes. Encoding fidelity also influences synchronization with different stimuli within each modality. Hove et al. (2013) found that in the auditory modality, synchronized tapping is more accurate with beeps than with continuous "sirens," likely because encoding of beeps is more temporally precise. In the visual modality, synchronized tapping is more accurate with moving stimuli than with flashes, likely resulting from the visual system's precise encoding of motion. "Modality appropriate" stimuli and stable tapping were associated with greater fMRI activation in the basal ganglia, a brain region implicated in motor control and temporal processing. These data suggest that precise encoding of the sensory target improves temporal processing in the basal ganglia and related subcorticocortical networks, thereby contributing to stable synchronization (Hove et al., 2013). Tierney and Kraus (2013b) contribute more direct evidence for the importance of sensory-encoding fidelity in synchronized tapping using brainstem recordings.

A connection between synchronized movements and sensory encoding has implications for basic neuroscience and may 
inform therapeutic interventions. Adolescents' tapping ability has been linked to higher-level cognitive functions, including attention and reading skills (Tierney and Kraus, 2013a). Tierney and Kraus (2013b) suggest that synchronized tapping and reading skills relate because both rely on consistent auditory brainstem timing. Thus, training that emphasizes beat synchronization could lead to more stable neural representation of sound, and improve cognitive and linguistic skills. The cross-domain links identified by the authors offer an intriguing starting point for speculation concerning causal relations. However, establishing causal relations requires future work.

Whether sensory-encoding consistency indeed causes individual differences in synchronized tapping as Tierney and Kraus (2013b) propose is currently unclear. Auditory encoding might not solely differentiate "good" and "poor" synchronization ability. As noted, synchronization relies on interconnected factors such as error correction and temporal prediction that are not assessed in the current setup. Consequently, the conceptual treatment and quantification of the ability to move to a beat in this context may not capture the complexity of sensorimotor synchronization. A straightforward interpretation that focuses on sensory encoding hence downplays the complexities of sensorimotor synchronization and risks narrowing the discussion to this particular factor. In the following, we develop this cautionary flag and provide a number of alternatives intended to extend the scope of the discussion as well as the spectrum for future research.

A bottom-up account is tempting when interpreting the tapping-cABR correlation, as low-level encoding occurs early in the sensorimotor loop. However, it is not entirely clear how small phaseencoding inconsistencies in the brainstem would expand by orders of magnitude to the levels of temporal variability in synchronized tapping. The comparably high variability in synchronized tapping might reflect a summation of "noise" throughout the perception-action cycle, i.e., several subprocesses contribute some variability. Hence, a more encompassing approach could attempt to isolate relative contributions to synchronized tapping variability. For example, does cABR consistency relate to variability in unpaced tapping, a measure previously used by the authors that is independent from encoding auditory targets (Tierney and Kraus, 2013a)?
Correlations between variability in unpaced tapping and brainstem encoding would suggest more noise throughout the nervous system, whereas if brainstem encoding correlated only with synchronized tapping, this would more strongly support the specific contribution of sensory encoding to synchronized tapping. Such an additional measure could hint at the relative contribution of more central temporal processing and help differentiate subprocesses needed for synchronization, e.g., by comparing "stable tappers" to "good correctors."

Synchronized tapping requires error correction and perception-action integration. Tierney and Kraus (2013b) suggest that auditory encoding precision could improve synchronized tapping via improved detection of tap-to-tone phase errors, and that future work might reveal a correlation between brainstem encoding consistency and lower phase-error detection thresholds. They mention that phase correction occurs in response to metronome shifts as small as $1.5 \mathrm{~ms}$. But one must note that $1.5 \mathrm{~ms}$ is the smallest shift tested, and the concept of "detection thresholds" might be immaterial. Considerable evidence indicates that phase correction does not involve extracting phase errors or asynchronies; instead, the timing of the next tap is based on a mixture (or weighted average) of timing from the preceding tone and the preceding tap (Repp, 2005; Repp and Su, 2013). Thus, looking for detection thresholds might be misguided. That said, phase correction and perception-action integration inevitably influence sensorimotor-synchronization variability, and understanding their neurophysiological underpinnings is important.

Another critical factor to consider when interpreting the results of the tapping and brainstem experiments is the role of top-down temporal prediction. The auditory stimuli in both experiments were isochronous and fully predictable, and accordingly would recruit similar top-down temporal prediction. Predictive mechanisms may influence measures of tapping variability and brainstem encoding, and thus could contribute to the reported correlation. In tapping, people predict the beat (and tap slightly before the onset; Repp, 2005); precise temporal prediction of the beat would decrease synchronized tapping variability. In the brainstem encoding experiment, accurate temporal prediction of syllable onset (over 6000 repetitions) would improve tuning and encoding consistency. Hence, brainstem-response variability might arise from poor prediction and inadequate topdown feedback to lower-level brainstem encoding (Bell et al., 2008). Such higherlevel processing and sensory tuning may involve dedicated temporal processing mechanisms and a brain network, including the basal ganglia and cerebellum, that can explicitly represent the temporal structure of an auditory sequence (Schwartze and Kotz, 2013).

In a similar vein, top-down prediction could account for the fact that tapping correlated with sensory encoding only during the initial formant transition. Prediction might more strongly influence encoding of this initial portion, which contains a perceptually relevant event: the so-called p-center. This subjective point of occurrence for speech segments relates to the onset of the first formant, i.e., an increase in energy around 500-1500 Hz (for a recent discussion, see Scott and McGettigan, 2012). In parallel, people align their taps relative to the p-center, which may provide salient timing information (Scott et al., 2009). Variable encoding of this critical information should compromise the ability to use temporal structure in perception and production, and lead to suboptimal behavior (Schwartze and Kotz, 2013).

In conclusion, the correlation between synchronized tapping and brainstem encoding reliability reported by Tierney and Kraus (2013b) provides stimulating new evidence for the neural underpinnings of individual differences in sensorimotor synchronization. Like most research, though, it supplies more questions than answers. Sensorimotor synchronization is a vibrant research area precisely because it requires integrating numerous processes across distributed neural systems. Ascribing central focus to bottom-up sensory encoding diminishes its richness and marginalizes other key processes such as top-down prediction. Accordingly, effective beat-synchronization interventions may enhance cognitive skills due in part to improved integration across distributed systems and/or improved prediction. To better understand the specific neural contributions of tapping with a beat and its consequences, supplementary multimethod investigations using neurophysiological methods and a battery of perceptual and motor tasks will prove critical. 


\section{References}

Bell CC, Han V, Sawtell NB (2008) Cerebellumlike structures and their implications for cerebellar function. Annu Rev Neurosci 31:1-24. CrossRef Medline

Hove MJ, Fairhurst MT, Kotz SA, Keller PE (2013) Synchronizing with auditory and visual rhythms: an fMRI assessment of modality differences and encoding reliabil-

ity. Neuroimage 67:313-321. CrossRef Medline

Repp BH (2005) Sensorimotor synchroniza- tion: a review of the tapping literature. Psychon Bull Rev 12:969-992. Medline

Repp BH, Su YH (2013) Sensorimotor synchronization: a review of recent research (2006-2012). Psychon Bull Rev 20:403-452. CrossRef Medline

Schwartze M, Kotz SA (2013) A dual-pathway neural architecture for specific temporal prediction. Neurosci Biobehav Rev 37:25872596. CrossRef Medline

Scott S, McGettigan C (2012) Amplitude onsets and spectral energy in perceptual experience. Front Psychol 3:80. CrossRef Medline
Scott SK, McGettigan C, Eisner F (2009) A little more conversation, a little less actioncandidate roles for the motor cortex in speech perception. Nat Rev Neurosci 10: 295-302. CrossRef Medline

Tierney A, Kraus N (2013a) The ability to tap to a beat relates to cognitive, linguistic, and perceptual skills. Brain Lang 124:225-231. CrossRef Medline

Tierney A, Kraus N (2013b) The ability to move to a beat is linked to the consistency of neural responses to sound. J Neurosci 33:1498114988. CrossRef Medline 